\title{
Beneficial effect of Mentha suaveolens essential oil in the treatment of vaginal candidiasis assessed by real-time monitoring of infection
}

\author{
Donatella Pietrella ${ }^{1}$, Letizia Angiolella ${ }^{2}$, Elisabetta Vavala², Anna Rachini ${ }^{1}$, Francesca Mondello ${ }^{3}$, Rino Ragno ${ }^{4}$,
} Francesco Bistoni ${ }^{1}$, Anna Vecchiarelli $i^{*}$

\begin{abstract}
Background: Vaginal candidiasis is a frequent and common distressing disease affecting up to $75 \%$ of the women of fertile age; most of these women have recurrent episodes. Essential oils from aromatic plants have been shown to have antimicrobial and antifungal activities. This study was aimed at assessing the anti-fungal activity of essential oil from Mentha suaveolens (EOMS) in an experimental infection of vaginal candidiasis.
\end{abstract}

Methods: The in vitro and in vivo activity of EOMS was assessed. The in vitro activity was evaluated under standard CLSI methods, and the in vivo analysis was carried out by exploiting a novel, non-invasive model of vaginal candidiasis in mice based on an in vivo imaging technique.

Differences between essential oil treated and saline treated mice were evaluated by the non-parametric MannWhitney U-test. Viable count data from a time kill assay and yeast and hyphae survival test were compared using the Student's t-test (two-tailed).

Results: Our main findings were: i) EOMS shows potent candidastatic and candidacidal activity in an in vitro experimental system; ii) EOMS gives a degree of protection against vaginal candidiasis in an in vivo experimental system.

Conclusions: This study shows for the first time that the essential oil of a Moroccan plant Mentha suaveolens is candidastatic and candidacidal in vitro, and has a degree of anticandidal activity in a model of vaginal infection, as demonstrated in an in vivo monitoring imaging system. We conclude that our findings lay the ground for further, more extensive investigations to identify the active EOMS component(s), promising in the therapeutically problematic setting of chronic vaginal candidiasis in humans.

\section{Background}

Candida albicans is a major fungal pathogen of humans $[1,2]$ and a commensal organism of the gastrointestinal tract. In severely immunocompromised patients this fungus causes high morbidity and mortality. C. albicans is also the etiological agent of vulvovaginal candidiasis, a common pathological condition, afflicting normal women of fertile age, which frequently develops into a chronic, substantially incurable, disease [3].

\footnotetext{
* Correspondence: vecchiar@unipg.it

${ }^{1}$ Microbiology Section, Department of Experimental Medicine and Biochemical Sciences, University of Perugia, Via del Giochetto, 06122 Perugia, Italy

Full list of author information is available at the end of the article
}

Different classes of antimycotic drugs are available to treat fungal infections. The azoles, particularly fluconazole, remain among the most common antifungal drugs, but their intensive clinical use for both therapy and prophylaxis has favoured the emergence of resistant strains [4]. The phenomenon of drug resistance has raised interest in substances of natural origin as a therapeutic alternative. Essential oils (EO) of aromatic plants are used by companies for the production of soaps, perfumes and toiletries. Many of them are also used in traditional medicine for various purposes [5-7]. In the last years various EO have been found to show antimicrobial, antioxidant anticancer and other pharmacological activities [8-10]. Particularly, a number of EO have been 
tested for in vivo and in vitro antimycotic activity and some have been shown to be potential antifungal agents.

The EO have a complex composition based on a number of constituents with low molecular weight, and their biological activities are due either to a main component of the mixture, usually a monoterpene, or to the synergic action of multiple compounds [11].

Mentha suaveolens has been used in the traditional medicine of Mediterranean areas and has a wide range of effects: tonic, stimulating, stomachic, carminative, analgesic, choleretic, antispasmodic, sedative, hypotensive and insecticidal. It shows depressor activity, analgesic and antiinflammatory action [12].

Mentha suaveolens plants collected in various regions of Morocco contains a high percentage of oxides such as piperitenone oxide (PEO) and piperitone oxide (PO), terpenic alcohol (fenohol, p-cymen-8-ol, geraniol, terpineol and borneol) and terpenic ketones (pulegone and piperitenone) all of which account for $65 \%$ to $90 \%$ of the total essential oil. The antimicrobial activity of PO, even if generally comparable to that of PEO, seems to be twofold lower than that of PEO against yeast [13]. No studies have however addressed the in vivo activity of Mentha suaveolens EO in a suitable experimental model of vaginal candidiasis under controlled conditions. Thus, in this study we have tested the in vitro and in vivo activity of M. suaveolens EO against C. albicans. Particularly, for in vivo activity, we used a recently developed, non-invasive in vivo imaging technique, which exploits a novel cell surface luciferase as reporter gene [14].

For both in vitro and in vivo studies, we used Jasmine Oil as a negative control and Tea Tree Oil as a positive control.

\section{Methods}

\section{Essential oils}

Mentha suaveolens essential oil was kindly provided by the Department of Chemistry and Drug Technologies, University of Rome "La Sapienza", Italy. It was obtained from wild-type plants grown in Tarquinia forests located around 60 miles from Rome. The oil was extracted by four-hour hydro distillation of the leaves using a Clevenger-type apparatus as previously described [15], then analyzed for chemical composition by gas chromatography and mass spectroscopy (DMePe BETA PS086, $0.25 \mathrm{~mm}$ film on a $25 \mathrm{~m}$ column, diameter of $0.25 \mathrm{~mm}$, operating at $220^{\circ} \mathrm{C}$ and eluting with helium). Compounds were identified by the application of the NIST 08 Mass Spectral Library. Analysis revealed that piperitenone oxide constitutes $90 \%$ of EOMS. Limonene and 1,8-cyneole were also present, among other minor constituents.

Essential oils of tea tree (Melaleuca alternifolia) (TTO) and jasmine oil (Jasminum grandiflorum) (JO) also used in this research were commercial oils purchased form Named (Lesmo, Italy) and Erboristeria Magentina (Torino, Italy), respectively. They were obtained by steam distillation from leaves and young branches of tea tree, and from flowers of jasmine. TTO is pure, extracted without additives and was used as a positive control, because of documented antifungal activity $[16,17]$ while jasmine oil, which was shown to be inactive against fungal growth, was used as a negative control [18].

Fluconazole was obtained from Sigma-Aldrich (Germany).

\section{Microorganisms}

Different strains of Candida albicans were used in the study: four clinical isolates from AIDS patients AIDS68, AIDS6, AIDS37 and AIDS126, CO23 isolated from a subject with vulvo-vaginal candidiasis susceptible to micafungin and fluconazole and the drug-resistant strains CO23RFK (micafungin-resistant) and CO23RFLU (fluconazole-resistant) [19], CA2, an echinocandin-resistant, non-germinative strain that grows as a pure yeast form at $28-37^{\circ} \mathrm{C}$ in conventional mycologic media [20], GR5 isolated from a woman with recurrent vaginal candidiasis, 3153 intrinsically resistant to fluconazole, ATCC10231 and ATCC24433. C. albicans CA1398 carrying the ACT1p-gLUC59 fusion (C. albicans gLUC59) or C. albicans CA1398 that did not express gLUC59 (control strain) were used in the models of vaginal Candida infections [14]. For experimental infections, cells from stock cultures in YPD agar (1\% yeast extract, $2 \%$ peptone, $2 \%$ glucose, $1.5 \%$ agar, all w/v) with $50 \mu \mathrm{g} / \mathrm{ml}$ chloramphenicol were grown in YPD broth ( $1 \%$ yeast extract, $2 \%$ peptone, $2 \%$ glucose, all w/v) at room temperature for $24 \mathrm{~h}$, then harvested by centrifugation, washed, counted in an haemocytometer, and resuspended to the desired concentration in sterile physiological saline. In order to examine the effect of the oil on the mycelia form of Candida, yeasts were grown for $4 \mathrm{~h}$ in RPMI 1640 plus $10 \% \mathrm{FBS}$ at $37^{\circ} \mathrm{C}$, then hyphae were washed and incubated with different concentrations of essential oils (EOMS, TTO and JO) for $24 \mathrm{~h}$ at $37^{\circ} \mathrm{C}$. Yeasts for infection were harvested from overnight cultures in YPD agar plates and adjusted to the concentration $10^{9} / \mathrm{ml}$ in sterile physiological saline.

\section{Minimal Inhibitory Concentration (MIC) assay}

The Minimal Inhibitory Concentration (MIC) was determined by micro-broth dilution method according to the Clinical and Laboratory Standards Institute/National Commitee for Clinical Laboratory Standards (CLSI/ NCCLS) Approved Standard M27-A3, 2008 [21]. Fluconazole $0.5 \mathrm{~g} / \mathrm{L}$ solution was prepared by dissolving the agent in endotoxin free water. Solutions of essential oils (100 g/L) were prepared in RPMI1640. Briefly, to 
determine the MIC of EOMS, TTO, JO or Fluconazole, RPMI-1640 supplemented with MOPS at $\mathrm{pH} 7$ was used. EOMS, TTO and JO were diluted in RPMI-1640 supplemented with Tween 80 (final concentration of $0.001 \% \mathrm{v} / \mathrm{v}$ ). The dilutions, ranging from 0.01219 to $12.48 \mathrm{~g} / \mathrm{L}$ of the essential oils, were prepared in 96 well plates. The inoculum size was about $2.5 \times 10^{3} \mathrm{cells} / \mathrm{ml}$. The plates were incubated at $30^{\circ} \mathrm{C}$ for $24-48 \mathrm{~h}$. To determine the hyphae survival, $C$. albicans cells were first grown for $4 \mathrm{~h}$ in RPMI supplemented with $10 \%$ of FBS serum and then treated with different essential oils.

\section{Minimal Fungicidal Concentration (MFC) assay}

The Minimal Fungicidal Concentration (MFC) was determined as the lowest concentration of Fluconazole or essential oils at which no microbial growth was observed. For the MFC determination, Sabouraud dextrose agar plates were seeded with $10 \mu \mathrm{l}$ of cell suspensions taken from the wells of the plates of MIC assay where cell growth was not observed. These plates were incubated at $30^{\circ} \mathrm{C}$ for $24-48 \mathrm{~h}$ and colony forming units (CFU) growth was evaluated.

\section{Time killing}

To confirm the fungicidal activity of EOMS, time-kill procedures were performed as described by Klepser [22] Cells sub-cultured in YPD at $28^{\circ} \mathrm{C}$ for $24 \mathrm{~h}$ were centrifuged, washed and resuspended at a concentration of $2.5 \times$ $10^{5} \mathrm{cell} / \mathrm{ml}$ in RPMI supplemented with EOMS or TTO and incubated at $28^{\circ} \mathrm{C}$. Essential oil concentrations used in the test were equivalent to $1,2,4$, and 8 times the MIC. At predetermined time points $(0,0.5,1,2,4,6,8,24$ and 48 hours) of incubation, $100 \mu \mathrm{l}$ aliquots were removed from the test solution and tenfold serial dilutions were performed. $100 \mu \mathrm{l}$ aliquot from each dilution was spread on the surface of Sabouraud dextrose agar plates and incubated at $37^{\circ} \mathrm{C}$ for $48 \mathrm{~h}$ for determination of $\mathrm{CFU} / \mathrm{ml}$.

\section{Cell lines}

Monomac-6, a human tumour cell line which was initially obtained from peripheral blood of a 60-year-old man with acute monocytic leukaemia, and L929, a fibroblastlike cell line cloned from strain L (the parent strain was derived from normal subartaneous areolar and adipose tissue of a male $\mathrm{C} 3 \mathrm{H} /$ An mouse) were grown in a humidified atmosphere containing $5 \%$ of $\mathrm{CO}_{2}$ at $37^{\circ} \mathrm{C}$. The culture medium consisted of RPMI 1640 with glutamine, $10 \%$ FBS (foetal bovine serum) and antibiotics. Every three or four days the cultures were split.

\section{Cytotoxicity assay}

The cytotoxicity was tested by the determination of the cell ATP level by ViaLight ${ }^{\circledR}$ Plus Kit (Lonza). The method is based upon the bioluminescent measurement of ATP that is present in all metabolically active cells. The bioluminescent method utilizes an enzyme, luciferase, which catalyses the formation of light from ATP and luciferin. The emitted light intensity is linearly related to the ATP concentration and is measured using a luminometer. To perform cytotoxicity tests, cells were recovered and counted and adjusted to the concentration $10^{6} / \mathrm{ml}$. The examinations were carried out for essential oils (EOMS, TTO and JO) and the control (cells not treated). Various 1:2 dilutions of the above mentioned oils were prepared in the medium (RMPI $1640,10 \%$ FBS, antibiotics) in order to achieve final concentrations in the wells: 1000-500-250-125-62.5-31-168-4-2-1-0 mg/L. Each concentration was tested in triplicate. After adding oils into appropriate wells, cells were added to each well to obtain the concentration of $10^{5}$ cells/well and incubated for $2 \mathrm{~h}$ at $37^{\circ} \mathrm{C}$. Plates were left in a room temperature to cool for 10 minutes and then the Cell Lysis Reagent was added to each well to extract ATP form the cells. Next, after 10 minutes the AMR Plus (ATP Monitoring Reagent Plus) was added and after 2 more minutes the luminescence was read using a microplate luminometer (TECAN).

\section{Mice}

Female CD1 mice obtained from Harlan Italy Laboratories (Udine, Italy) were used at 4 to 6 weeks of age. Mice were allowed to rest for 1 week before the experiment; by that time the animals were roughly 5 to 7 weeks old. Animals were used under specific-pathogenfree conditions that included testing sentinels for unwanted infections; according to the Federation of European Laboratory Animal Science Association standards, no infections were detected.

The experimental research was approved on 25 January 2008 by the Ethics Committee of the University of Perugia.

\section{Infection and treatment}

Mice infection was performed as previously described with minor adaptations [23]. Mice were maintained under pseudoestrus condition by subcutaneous injection of $0.2 \mathrm{mg}$ of estradiol valerate in $100 \mu \mathrm{l}$ of sesame oil (Sigma-Aldrich) 6 days prior to infection and weekly until the completion of the study. Mice anaesthetized with 2.5-3.5 (v/v) isofluorane gas were infected twice at a $24 \mathrm{~h}$ interval with $10 \mu \mathrm{l}$ of $10^{9} \mathrm{cell} / \mathrm{ml}$ of C. albicans gLUC59 or the control strain. Cell suspensions were administered from a mechanical pipette into the vaginal lumen, close to the cervix. To favour vaginal contact and adsorption of fungal cells, mice were held head down for $1 \mathrm{~min}$ following inoculation. Mice were then allowed to recover for 24-48 h, during which the Candida infection was established. 
The intravaginal treatment with TTO, EOMS and JO (500 $\mu \mathrm{g} / 10 \mu \mathrm{l} /$ mouse) was begun $2 \mathrm{~h}$ before the first challenge and then it was repeated every two days until day +21 .

\section{Monitoring of mouse vaginal infection}

To monitor the infection during the treatment with essential oil, every day post-infection (starting $48 \mathrm{~h}$ after challenge) $10 \mu \mathrm{l}\left(1 \mathrm{mg} / \mathrm{ml}\right.$ in 1:4 methanol: $\left.\mathrm{H}_{2} \mathrm{O}\right)$ of coelenterazine was added to the vaginal lumen. Afterwards, mice were imaged in the IVIS-200TM imaging system under anaesthesia with $2.5 \%$ isoflurane. Total photon emission from vaginal areas within the images (Region Of Interest, ROI) of each mouse was quantified with Living ImageR software package. In selected experiments mice were anaesthetized with $2.5 \%$ isoflurane and then held head down, the vaginal lumen was thoroughly washed with $150 \mu \mathrm{l}$ of saline. To determine the fungal load in the vagina, $50 \mu \mathrm{l}$ of the lavage fluids from each mouse were plated on YPD agar plus chloramphenicol $(50 \mu \mathrm{g} / \mathrm{ml})$, then CFUs were evaluated.

\section{Statistical analysis}

Differences between essential oil treated and saline treated mice were evaluated by the non-parametric MannWhitney U-test. Viable count data from time kill assay and yeast and hyphae survival test were compared using the Student's t-test (two-tailed). $P$-values of $<0.05$ were considered significant.

\section{Results}

MIC, MFC and Killing Kinetics

The initial determination of the antifungal activity of essential oils (EOMS, TTO and JO) was performed in vitro by standardized CLSI/NCCLS methods[21] and this was done against all strains of C. albicans used throughout this study. MIC values fell in a range of 0.39-0.78 g/L for EOMS and 0.78-3.12 g/L for TTO. The MFC values ranged from $0.39-1.56 \mathrm{~g} / \mathrm{L}$ for EOMS and 1.56-6.24 for TTO, thus very close to MIC values. In our experimental system, TTO was less efficient than EOMS especially when the oils were tested against fluconazole resistant strains. In addition MFC values for TTO were higher than EOMS for all strains tested (table 1). JO, used as a negative control, did not affect the growth of any strain tested.

To obtain more insight into the anticandidal activity of EOMS on gLUC59, i.e the strain used in the experimental vaginal infection (see below), a time-kill test at concentrations equivalent to $1,2,4$, and 8 times of the MIC value $(0.39 \mathrm{~g} / \mathrm{L})$ was performed. As reported in Figure 1 , at a concentration of $2 \times \mathrm{MIC}(0.78 \mathrm{~g} / \mathrm{L})$, the number of colonies was significantly reduced after 24 hrs of incubation $(P<0.05)$ and the total fungicidal effect was observed within 48 hrs of contact. The results demonstrated that C. albicans gLUC59 was highly susceptible to EOMS. In parallel we analyzed the time kill test for TTO, confirming that this oil exerted a fungicidal effect within $48 \mathrm{hrs}$ at a concentration higher $(3,12 \mathrm{~g} / \mathrm{L} ; P<0.05)$ than that observed with the EOMS $(0,78 \mathrm{~g} / \mathrm{L})$.

\section{Yeasts and the mycelial form have different} susceptibilities to EOMS

Given that EOMS affects C. albicans yeast forms of growth, we extended our investigations to the virulent mycelial form of C. albicans. To this end C. albicans was cultured at $37^{\circ} \mathrm{C}$ for $4 \mathrm{~h}$ in the presence of $10 \%$ of FBS serum. Microscopic examination demonstrated

Table 1 Antifungal activity of EOMS, TTO, JO and fluconazole on different Candida albicans strains

\begin{tabular}{|c|c|c|c|c|c|c|c|c|}
\hline \multirow[b]{2}{*}{ Strains } & \multicolumn{2}{|c|}{ EOMS } & \multicolumn{2}{|c|}{ TTO } & \multicolumn{2}{|c|}{ JO } & \multicolumn{2}{|c|}{ Fluconazole } \\
\hline & MIC g/L & MFC g/L & $\mathrm{MIC} g / \mathrm{L}$ & MFC g/L & MIC g/L & MFC g/L & $\mathrm{MIC} \mathrm{mg/L}$ & $\mathrm{MFC} \mathrm{mg} / \mathrm{L}$ \\
\hline AIDS68 & 0.39 & 0.78 & 3.12 & 3.12 & $>100$ & $>100$ & $>64$ & $>128$ \\
\hline AIDS6 & 0.39 & 0.78 & 3.12 & 6.24 & $>100$ & $>100$ & $>64$ & $>128$ \\
\hline AIDS37 & 0.39 & 0.39 & 3.12 & 6.24 & $>100$ & $>100$ & $>64$ & $>128$ \\
\hline AIDS126 & 0.39 & 0.78 & 3.12 & 6.24 & $>100$ & $>100$ & 0,25 & 2 \\
\hline $\mathrm{CA} 2$ & 0.39 & 0.39 & 3.12 & 6.24 & $>100$ & $>100$ & 0,5 & $>128$ \\
\hline GR5 & 0.39 & 0.78 & 3.12 & 3.12 & $>100$ & $>100$ & 2 & $>128$ \\
\hline 3153 & 0.39 & 0.78 & 3.12 & 6.24 & $>100$ & $>100$ & 64 & $>128$ \\
\hline $\mathrm{CO} 23$ & 0.39 & 0.39 & 0.78 & 1.56 & $>100$ & $>100$ & 0.25 & 1 \\
\hline CO23RFK & 0.78 & 1.56 & 0.78 & 1.56 & $>100$ & $>100$ & 0.25 & 1 \\
\hline CO23RFLU & 0.78 & 1.56 & 0.78 & 3.12 & $>100$ & $>100$ & $>64$ & $>128$ \\
\hline ATCC10231 & 0.39 & 0.39 & 3.12 & 6.24 & $>100$ & $>100$ & 4 & 4 \\
\hline ATCC24433 & 0.39 & 0.39 & 3.12 & 6.24 & $>100$ & $>100$ & 0.25 & 1 \\
\hline gLUC59 & 0.39 & 1.56 & 3.12 & 6.24 & $>100$ & $>100$ & 0.39 & 0.78 \\
\hline gLUC59 Control strain & 0.39 & 1.56 & 3.12 & 6.24 & $>100$ & $>100$ & 0.39 & 0.78 \\
\hline
\end{tabular}




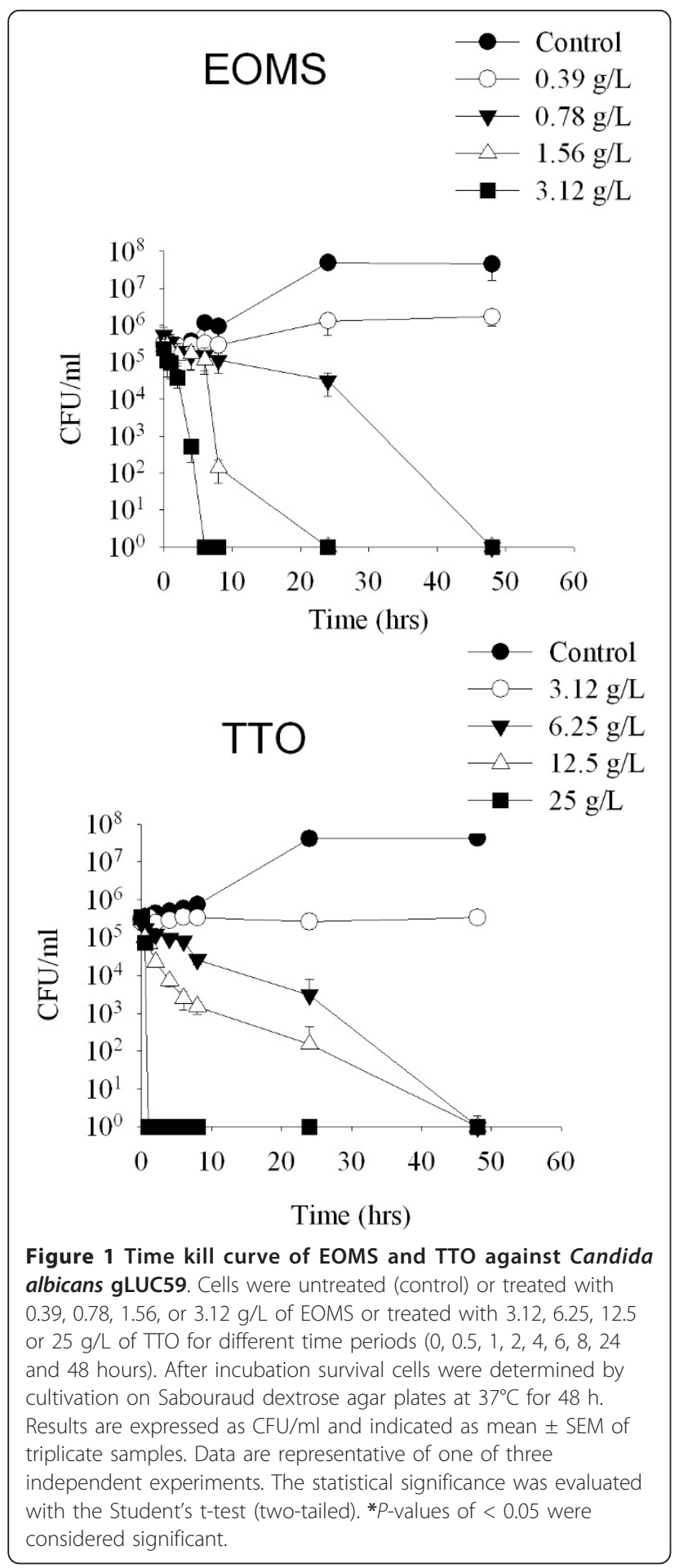

$>90 \%$ mycelial conversion under our conditions. As shown in Figure 2, hyphal forms were less susceptible to EOMS than yeast forms, since inhibition of hyphal growth is obtained at a significantly higher concentration $(0.098 \mathrm{~g} / \mathrm{L})$ than the concentration required to inhibit yeast forms of growth. In fact, a concentration of 0.5 $\mathrm{g} / \mathrm{L}$ was able to completely inhibit the yeast cells but not the hyphal form (Figure 2A-B) Notably, the EOMS showed a greater inhibitory effect than TTO, in both yeast cells ( 0.05 vs $0.098 \mathrm{~g} / \mathrm{L}$ respectively) and the hyphal form ( 0.098 vs $0.39 \mathrm{~g} / \mathrm{L}$ respectively). Jasmine Oil did not affect the viability of yeast or mycelial cells. The lack of effect of EOMS $0.5 \mathrm{~g} / \mathrm{L}$ on hyphal survival was documented by microscopic examination of hyphal damage observed after addition of EOMS (Figure 2C).

Experiments were also performed to examine whether the EOMS expressed cytotoxicity towards immune cells. To this end, Monomac 6 and L929 cell lines were treated with various concentrations of EOMS for 2 and 24 $h$. The results reported in Figure 3 show that high concentrations of EOMS (0.5 and $1 \mathrm{~g} / \mathrm{L})$, i.e. higher than the MIC value, were necessary to exert cytotoxicity on Monomac 6 and L929 cell lines. Using the same concentrations a similar trend was observed for TTO, while no cytotoxicity resulted from JO treatment.

\section{Effect of EOMS on vaginal candidiasis}

Given the encouraging results observed in vitro, we wondered whether these beneficial effects against $C$. albicans could be reproduced in an in vivo system. To this purpose we exploited the new in vivo imaging technique which we have recently developed in our laboratory $[14,24]$ to assess therapeutic efficacy in an estrogen-dependent mouse model of vaginal candidiasis.

Estradiol treated mice were infected with $C$. albicans expressing luciferase gene gLUC and EOMS, TTO or JO were administered intravaginally $2 \mathrm{~h}$ before the first challenge and then every two days. The course of the infection was monitored at various days post challenge by in vivo imaging (Figure 4). The fungal load in the vagina was quantified as photon emission as well as CFU from vaginal fluids. The results reported in Figure 5 show that there is a significant reduction of Candida load in mice treated with EOMS with respect to diluent (saline) treated mice starting from 15 days post infection, and this beneficial effect was maintained until 21 days post infection. In this model, and under the conditions tested, TTO was only minimally effective in causing a significant reduction of vaginal fungus load, measured as photon emission at 9 and 15 days. No effect was recorded after 21 days of infection.

\section{Discussion}

Human pathogenic fungi represent a significant proportion of the infectious agents affecting the immunocompromised host. The therapeutic options for these patients are hampered by i) the relative scarcity of active and safe antifungal drugs, most of which are essentially fungistatic rather than fungicidal, ii) antifungal drug resistance to the most active and widely used azole compounds, iii) the difficulties of devising and/or constantly 


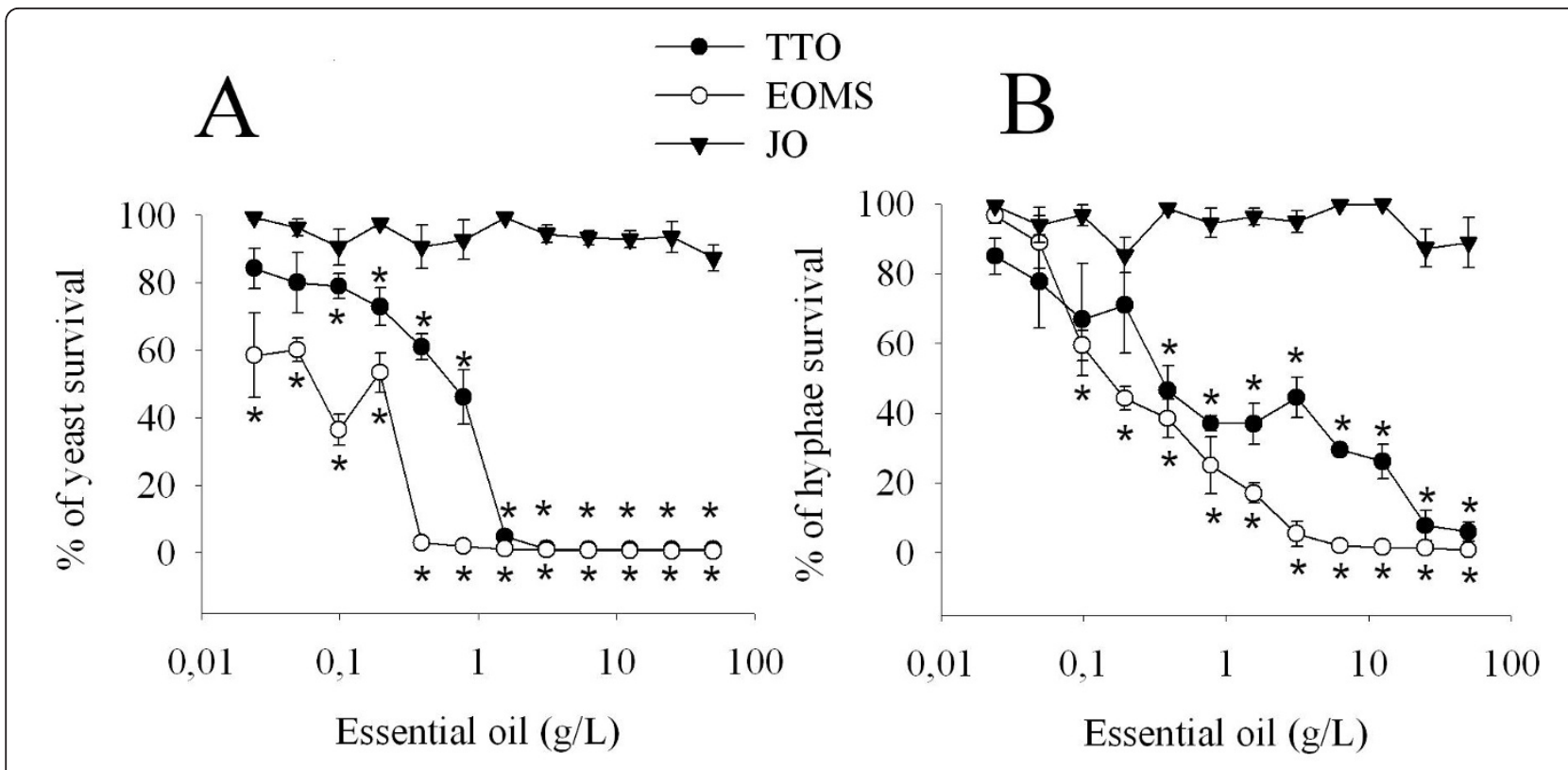

$\mathrm{C}$
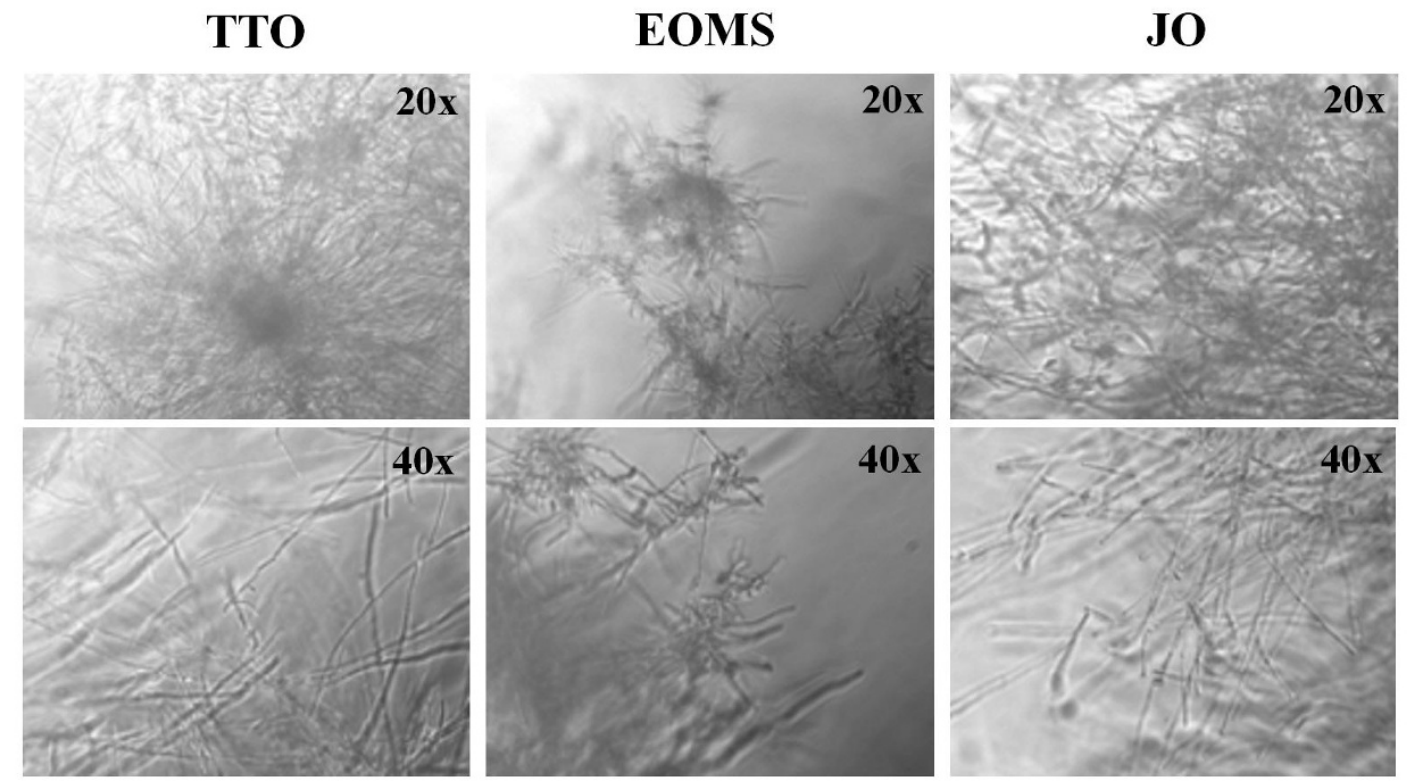

Figure 2 Effect of essential oil on yeast and hyphae survival. gLUC59 Candida albicans yeast cells (A) and preformed hyphae (B) were treated with different concentrations of essential oils (EOMS, TTO and JO) for $24 \mathrm{~h}$. After incubation $10 \mu \mathrm{l}$ of coelenterazine substrate (1 mg/ml) was added to each well and samples were read using a luminometer. Results are expressed as percentage of yeast or hyphae survival and indicated as mean \pm SD of triplicate samples are from one of three experiments with similar results. The statistical significance was evaluated with the Student's t-test (two-tailed). * $P$-values of $<0.05$ were considered significant. The effect on preformed hyphae was microscopically examined after $24 \mathrm{~h}$ of treatment with essential oil (C). Original magnification of 20x or 40x is indicated in the micrographs. The results are representative of one of three independent experiments.

maintaining effective infection control measures in the health care institutions. Overall, fungal infections in immunodepressed subjects are a very challenging problem for the health system.

Thus there is a clear demand for finding a new therapeutic approach in this era of increasing spreading of antimicrobial drug resistance and re-emergence of infectious diseases $[25,26]$.

Recently the use of TTO as a new approach in antifungal therapy has been proposed. This natural compound appears to be effective in vitro against multidrug resistant Candida and in vivo against mucosal candidiasis [27]. 


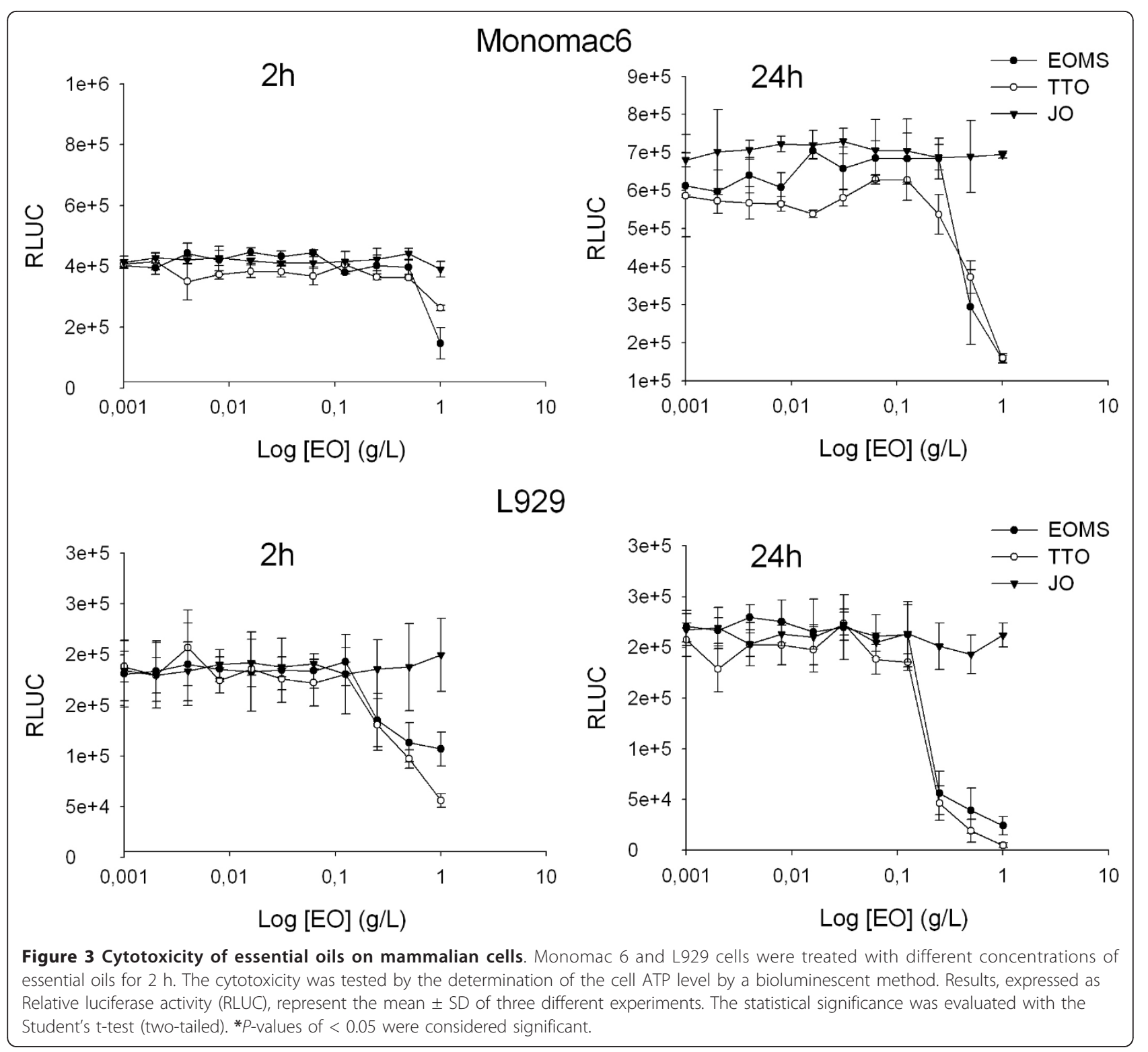

Moreover it has also been documented that terpinen-4-ol rather than 1,8-cineole is the most likely mediator of TTO activity or, at least, a main contributor to anti-Candida activity [16]. In this study we used TTO as a positive control in our in vitro and in vivo experimental system.

Regarding the antimicrobial properties of EOMS recent evidence attributes larvicidal activity to this essential oil and its active compound [28]. Other important activities of EOMS include protective effects against hydrogen-peroxide-induced-cytotoxicity. Anti-Candida activity has been described for Mentha piperita [29]. Furthermore EOMS was effective against Gram positive and Gram negative microorganisms and fungi [13]. The main microbicidal components of EOMS were pulegone and piperitone oxide.
In this study we demonstrated for the first time that EOMS is endowed with potent anticandidal activity in vitro, both against azole-susceptible and azole-resistant Candida strains. In addition, EOMS was shown to be not only an inhibitor of Candida growth, but also able to actually kill the yeasts. We determined the time killing curves, and so discovered that EOMS was apparently more effective than the more extensively investigated TTO. All experiments were performed against a control, the jasmine oil, which proved totally ineffective.

The antifungal activity is manifested against both yeast and the mycelial form, although higher EOMS concentrations were required to kill these latter forms of growth. Finally, we provide evidence that intravaginal administration of EOMS in vivo is also efficacious to some degree. 


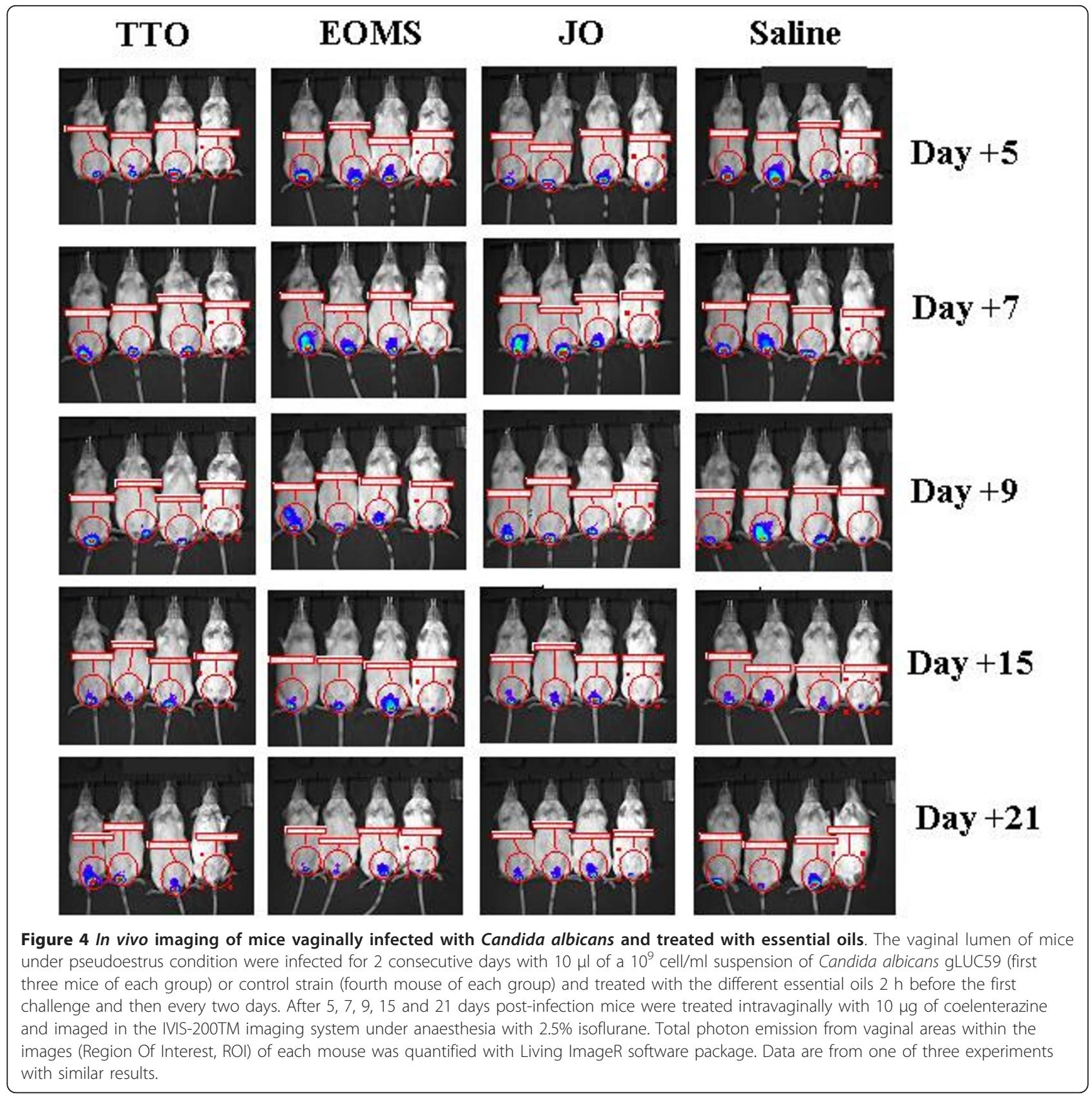

For the in vivo assay, a stringent and controlled model of vaginal infection of mice was used. This exploits a novel cell surface luciferase as reporter gene, constructed by fusing a synthetic, codon-optimized version of the Gaussia princeps luciferase gene to Candida albicans PGA59, which encodes a glycosylphosphatidyl inositol-linked cell wall protein [14]. This technique allows a continuous, non invasive monitoring of the spatial and temporal progression of vaginal infection in a small number of live mice. The model proved useful in assaying for anticandidal protection in actively or passively immunized animals [24]. The method was paralleled by a more traditional determination of vaginal fungus load in the vagina by $\mathrm{CFU}$. The in vivo imaging technique resulted much more sensitive than the classic CFU method for at least two different reasons: 1) the vaginal wash doesn't completely clear the vaginal lumen because the Candida hyphae are well attached to the tissue; 2) several hyphae often grew as a single colony, causing an underestimation of the fungal load.

Overall, we show here that EOMS accelerates the clearance of fungus during vaginal candidiasis, and this 


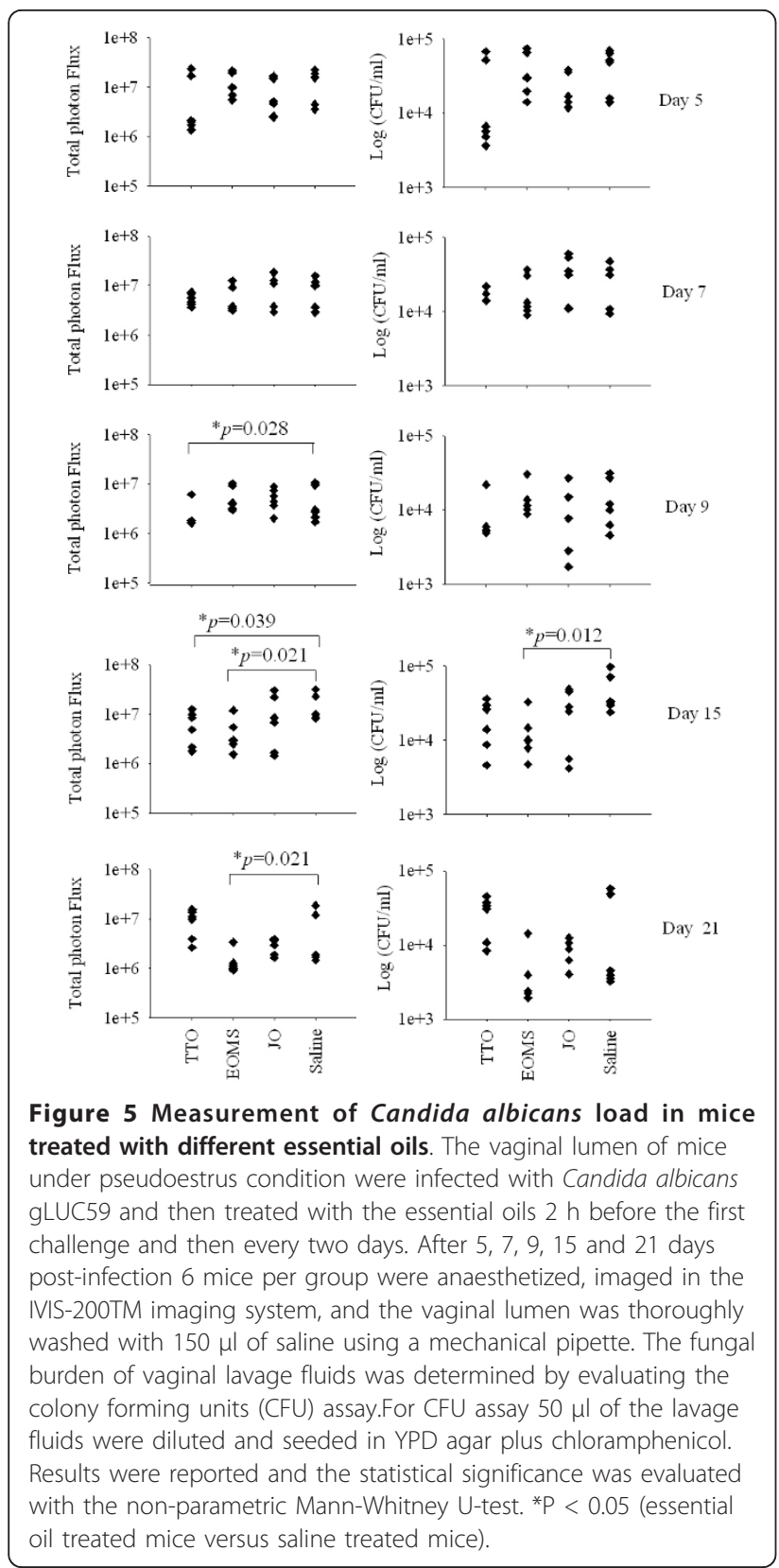

accelerated clearance of Candida is demonstrated by both photon emission and CFU measurements. The EOMS activity in our model seems superior, at least after 21 days of infection, to that of TTO, which has previously been found particularly efficacious in a rat model of vaginal candidiasis [16].

Our data are potentially relevant in the treatment of Candida vulvovaginal infection (VVC). This is a frequent and commonly distressing disease affecting $70-75 \%$ of childbearing age women worldwide at least once during their lives. Predisposing factors for developing an acute form of vaginal candidiasis include antibiotic and oral contraceptive usage, hormone replacement therapy, pregnancy, uncontrolled diabetes mellitus and African American ethnicity [30,31]. $5 \%$ and possibly up to $10 \%$ of women with a primary episode subsequently experience frustrating recurrent VVC (RVVC) which is defined as at least three-four specific episodes within one year [3,32].

\section{Conclusions}

This study shows for the first time that: i) EOMS has considerable in vitro, candidastatic and candidacidal activity ii) EOMS administration in vivo accelerates the clearance of $C$. albicans during vaginal infection.

The high impact of this infection and the difficulty of finding an effective therapy reinforces the need to search for an alternative therapeutic approach to integrate or even replace the current treatment. The present results could provide the ground for further investigations, particularly aimed at identifying the therapeutically active anticandidal EOMS component(s).

\section{Acknowledgements}

We thank Professor Antonio Cassone for critical reading of the paper. We are grateful to Catherine Macpherson for editorial assistance.

This work was supported by a grant from the European Commission [FINSysB Marie Curie Initial Training Network, PITNGA-2008-214004] and by a grant from Fondazione Cassa Risparmio Perugia 2009.010.0492.

\section{Author details}

${ }^{1}$ Microbiology Section, Department of Experimental Medicine and Biochemical Sciences, University of Perugia, Via del Giochetto, 06122 Perugia, Italy. ${ }^{2}$ Department of Public Health and Infectious Diseases, University of Rome 'La Sapienza', Piazzale Aldo Moro, 00185 Rome, Italy. ${ }^{3}$ Department of Infectious, Parasitic and Immune-mediated Diseases, Istituto Superiore di Sanità, Viale Regina Elena 299, 00161 Rome, Italy. ${ }^{4}$ Department of Chemistry and Drug Technologies, University of Rome 'La Sapienza', Piazzale Aldo Moro, 00161 Rome, Italy.

\section{Authors' contributions}

$D P, A R$ carried out the in vivo experiments and part of the MIC evaluation. LA, EV, FM, RR carried out the essential oil extraction, the MIC and time killing curve experiments. FB participated in the design and coordination of the study. AV conceived of the study and was primarily involved in the conceptual planning of the paper. All authors read and approved the final manuscript.

\section{Competing interests}

A patent related to piperitenone oxide, the main component of Mentha suaveolens essential oil, and its possible industrial application, has been filed by $L A$ and $R R$.

Received: 31 August 2010 Accepted: 28 February 2011 Published: 28 February 2011

\section{References}

1. Calderone RA, (ed): Candida and Candidiasis. Washington, D.C.: ASM Press; 2002.

2. Odds FC, (ed): Candida and Candidosis. London, United Kingdom: Bailliere Tindall; 21988.

3. Sobel JD: Vulvovaginal candidosis. Lancet 2007, 369(9577):1961-1971.

4. Sullivan DJ, Moran GP, Pinjon E, Al-Mosaid A, Stokes C, Vaughan C, Coleman DC: Comparison of the epidemiology, drug resistance mechanisms, and virulence of Candida dubliniensis and Candida albicans. FEMS Yeast Res 2004, 4(4-5):369-376.

5. Cowan MM: Plant products as antimicrobial agents. Clin Microbiol Rev 1999, 12(4):564-582. 
6. Stockwell C, (ed): Nature's pharmacy. London, UK: Century Hutchinson Ltd; 1988.

7. Thomson WAR, (ed): Medicines from Hearth. Maidenhead, UK: McGraw-Hill Book Co; 1978.

8. Arweiler NB, Donos N, Netuschil L, Reich E, Sculean A: Clinical and antibacterial effect of tea tree oil-a pilot study. Clin Oral Investig 2000, 4(2):70-73.

9. Calcabrini A, Stringaro A, Toccacieli L, Meschini S, Marra M, Colone M, Salvatore G, Mondello F, Arancia G, Molinari A: Terpinen-4-ol, the main component of Melaleuca alternifolia (tea tree) oil inhibits the in vitro growth of human melanoma cells. J Invest Dermatol 2004, 122(2):349-360.

10. Martin KW, Ernst E: Herbal medicines for treatment of fungal infections: a systematic review of controlled clinical trials. Mycoses 2004, 47(3-4):87-92.

11. Bakkali F, Averbeck S, Averbeck D, Idaomar M: Biological effects of essential oils-a review. Food Chem Toxicol 2008, 46(2):446-475.

12. Moreno L, Bello R, Primo-Yufera E, Esplugues J: Pharmacological properties of the methanol extract from Mentha suaveolens Ehrh. Phytother Res 2002, 16(Suppl 1):S10-13.

13. Oumzil H, Ghoulami S, Rhajaoui M, llidrissi A, Fkih-Tetouani S, Faid M, Benjouad A: Antibacterial and antifungal activity of essential oils of Mentha suaveolens. Phytother Res 2002, 16(8):727-731.

14. Enjalbert B, Rachini A, Vediyappan G, Pietrella D, Spaccapelo R, Vecchiarelli A, Brown AJ, d'Enfert C: A multifunctional, synthetic Gaussia princeps luciferase reporter for live imaging of Candida albicans infections. Infect Immun 2009, 77(11):4847-4858.

15. Angiolella LVE, Sivric S, Diodata D'AF, Ragno R: In vitro activity of Mentha suaveolens essential oil against Cryptococcus neoformans and dermatophytes. International Journal of Essential Oil Therapeutics 2010, 4(1-2):35-36

16. Mondello F, De Bernardis F, Girolamo A, Cassone A, Salvatore G: In vivo activity of terpinen-4-ol, the main bioactive component of Melaleuca alternifolia Cheel (tea tree) oil against azole-susceptible and -resistant human pathogenic Candida species. BMC Infect Dis 2006, 6:158.

17. Rosato A, Vitali C, Gallo D, Balenzano L, Mallamaci R: The inhibition of Candida species by selected essential oils and their synergism with amphotericin B. Phytomedicine 2008, 15(8):635-638.

18. Agarwal V, Lal P, Pruthi V: Prevention of Candida albicans biofilm by plant oils. Mycopathologia 2008, 165(1):13-19.

19. Angiolella L, Stringaro AR, De Bernardis F, Posteraro B, Bonito M, Toccacieli L, Torosantucci A, Colone M, Sanguinetti M, Cassone A, et al: Increase of virulence and its phenotypic traits in drug-resistant strains of Candida albicans. Antimicrob Agents Chemother 2008, 52(3):927-936.

20. Cassone A, Mason RE, Kerridge D: Lysis of growing yeast-form cells of Candida albicans by echinocandin: a cytological study. Sabouraudia 1981, 19(2):97-110

21. NCCLS (ed): National Committee for Clinical Laboratory Standards. Methods for broth dilution antifungal susceptibility testing of yeast. Villanova, Pa., USA: NCCLS documents; "Thirty 2008, 27-A3, approved standard edn.

22. Klepser ME, Ernst EJ, Lewis RE, Ernst ME, Pfaller MA: Influence of test conditions on antifungal time-kill curve results: proposal for standardized methods. Antimicrob Agents Chemother 1998 42(5):1207-1212.

23. Fidel PL Jr, Lynch ME, Sobel JD: Candida-specific Th1-type responsiveness in mice with experimental vaginal candidiasis. Infect Immun 1993, 61(10):4202-4207.

24. Pietrella D, Rachini A, Torosantucci A, Chiani P, Brown AJ, Bistoni F, Costantino P, Mosci P, d'Enfert C, Rappuoli R, et al: A beta-glucanconjugate vaccine and anti-beta-glucan antibodies are effective against murine vaginal candidiasis as assessed by a novel in vivo imaging technique. Vaccine 2010, 28(7):1717-1725.

25. Levy SB, Marshall B: Antibacterial resistance worldwide: causes, challenges and responses. Nat Med 2004, 10(12 Suppl):S122-129.

26. Perea S, Patterson TF: Antifungal resistance in pathogenic fungi. Clin Infect Dis 2002, 35(9):1073-1080.

27. Mondello F, De Bernardis F, Girolamo A, Salvatore G, Cassone A: In vitro and in vivo activity of tea tree oil against azole-susceptible and -resistant human pathogenic yeasts. J Antimicrob Chemother 2003, 51(5):1223-1229.

28. Koliopoulos G, Pitarokili D, Kioulos E, Michaelakis A, Tzakou O: Chemical composition and larvicidal evaluation of Mentha, Salvia, and Melissa essential oils against the West Nile virus mosquito Culex pipiens. Parasitol Res 2010

29. Yigit D, Yigit N, Ozgen U: An investigation on the anticandidal activity of some traditional medicinal plants in Turkey. Mycoses 2009, 52(2):135-140.

30. Cassone A, De Bernardis F, Santoni G: Anticandidal immunity and vaginitis: novel opportunities for immune intervention. Infect Immun 2007, 75(10):4675-4686.

31. Xu J, Schwartz K, Bartoces M, Monsur J, Severson RK, Sobel JD: Effect of antibiotics on vulvovaginal candidiasis: a MetroNet study. J Am Board Fam Med 2008, 21(4):261-268.

32. Magliani W, Conti S, Cassone A, De Bernardis F, Polonelli L: New immunotherapeutic strategies to control vaginal candidiasis. Trends $\mathrm{Mol}$ Med 2002, 8(3):121-126.

\section{Pre-publication history}

The pre-publication history for this paper can be accessed here: http://www.biomedcentral.com/1472-6882/11/18/prepub

doi:10.1186/1472-6882-11-18

Cite this article as: Pietrella et al:: Beneficial effect of Mentha suaveolens essential oil in the treatment of vaginal candidiasis assessed by realtime monitoring of infection. BMC Complementary and Alternative Medicine 2011 11:18.

\section{Submit your next manuscript to BioMed Central and take full advantage of:}

- Convenient online submission

- Thorough peer review

- No space constraints or color figure charges

- Immediate publication on acceptance

- Inclusion in PubMed, CAS, Scopus and Google Scholar

- Research which is freely available for redistribution
Ciomed Central 